\title{
Impacto de la pandemia de la COVID-19 en el funcionamiento de un servicio público de Ortopedia y Traumatología: nuestro aprendizaje luego de la primera ola
}

\author{
Guillermo A. Ricciardi, Fernando Locaso, Martín A. Pérez, Santiago Soto Arriaga, Santiago Scalambro, Ariel A. Salinas, \\ Mariano Riva, Alan Elorza, Diego Mansilla, Florencia Molina, Juan Urrica, Gustavo Ricciardi, Sol Manterola, Ignacio G. \\ Garfinkel, Gabriel G. Carrioli, Daniel O. Ricciardi \\ Servicio de Ortopedia y Traumatología, Hospital General de Agudos “Dr. Teodoro Álvarez”, Ciudad Autónoma de Buenos Aires, Argentina
}

\begin{abstract}
RESUMEN
Introducción: El objetivo principal fue comparar la actividad clínico-quirúrgica de nuestro Servicio de Ortopedia y Traumatología antes de la instauración del plan de contingencia frente a la pandemia de la COVID-19 y durante este, un aspecto relevante para establecer antecedentes que permitan guiar nuestro desempeño ante la segunda ola de casos y futuras contingencias semejantes. Materiales y Métodos: Estudio analítico observacional longitudinal ambispectivo de los pacientes atendidos en el Servicio de Ortopedia y Traumatología de un hospital del sistema público de la Ciudad Autónoma de Buenos Aires, durante el plan de contingencia frente a la pandemia de la COVID-19. Como variable de resultado primaria se valoró el número total de cirugías realizadas durante el período de estudio. Resultados: La pandemia se asoció con menos cirugías totales $(p=0,002)$, de Guardia $(p=0,000)$ y de Planta $(p=0,002)$. Las cirugías totales se redujeron un $81,5 \%$. Las cirugías de urgencia representaron el $97 \%$, diferencia significativa con el período fuera de la pandemia $(p=0,080)$. Además, el promedio de cirugías por día quirúrgico disponible fue significativamente menor $(p=0,000)$. Conclusiones: Creemos que la atención de la enfermedad ortopédica y el trauma musculoesquelético, adaptada a las obligaciones indicadas por el plan nacional y regional de contingencia frente a la pandemia, fue notoriamente menor que nuestro desempeño habitual. Si bien respondimos adecuadamente a las urgencias de nuestra especialidad queda pendiente la posibilidad de estratificar y considerar tipos de pacientes con cuadros pasibles de ser tratados, en forma gradual, durante la pandemia.
\end{abstract}

Palabras clave: COVID-19; ortopedia y traumatología; epidemiología; funcionamiento.

Nivel de Evidencia: IV

Impact of the COVID-19 Pandemic on the Activity of a Public Orthopedics and Traumatology Service: Our Insights After the First Wave

\begin{abstract}
Introduction: Our aim was to compare our activity in the Orthopedics and Traumatology service during the first wave of the COVID-19 pandemic. Materials and Methods: Ambispective longitudinal observational analytical study of patients treated in the Orthopedics and Traumatology Service of a public health hospital of Buenos Aires during the first wave of the COVID-19 pandemic. The primary outcome variable was the total number of surgeries performed during the study period. Results: The pandemic was associated with fewer total surgeries $(p=0.002)$, emergency department surgeries $(p=0.000)$ and elective surgeries $(p=0.002)$. Total surgeries were reduced by $81.5 \%$. Emergency surgeries accounted for $97 \%$, a significant difference with the non-pandemic period $(p=0.080$ ). Additionally, the average number of surgeries per available surgical day was significantly lower $(p=0.000)$. Conclusions: We believe that, under the regulations indicated by the national and regional pandemic contingency plan, the care of orthopedic pathology and musculoskeletal trauma was notoriously lower than our usual performance. Although we responded adequately to the emergencies of our specialty, the possibility of stratifying and considering types of patients with conditions that could be treated gradually during the pandemic remains pending.
\end{abstract}

Key words: COVID-19; orthopaedics and traumatology; epidemiology; activity.

Level of Evidence: IV

Recibido el 9-4-2021. Aceptado luego de la evaluación el 8-8-2021 • Dr. GUILLERMO A. RICCIARDI • guillermoricciardi @ gmail.com ID https://orcid.org/0000-0002-6959-9301 Cómo citar este artículo: Ricciardi GA, Locaso F, Pérez MA, Soto Arriaga S, Scalambro S, Salinas AA, et al. Impacto de la pandemia de la COVID-19 en el funcionamiento de un servicio público de Ortopedia y Traumatología: nuestro aprendizaje luego de la primera ola. Rev Asoc Argent Ortop Traumatol 2021;86(6):716-726. https://doi.org/10.15417/issn.1852-7434.2021.86.6.1341 


\section{INTRODUCCIÓN}

La rápida propagación internacional del virus SARS-CoV-2 desde su detección original en Wuhan (diciembre de 2019) hasta la declaración del estado de pandemia por la Organización Mundial de la Salud en menos de tres meses, obligó a la rápida restructuración del funcionamiento de los sistemas de salud del mundo que impactó en la actividad de todas las áreas y especialidades que lo componen. ${ }^{1,2}$ En nuestro medio, se suma el agravante de carecer de un antecedente de magnitud comparable en la Argentina y en países de América Latina.

Bajo los lineamientos de un estado de emergencia sanitaria, los Servicios de Ortopedia y Traumatología debieron limitar y adaptar su actividad, condicionados por las diferentes etapas de la pandemia y con el objetivo fundamental de limitar el crecimiento exponencial de casos.

Las preocupaciones relacionadas con la COVID-19 invadieron nuestra práctica, fue necesario el esfuerzo mancomunado de todo el personal de salud para llevar a cabo los tres objetivos fundamentales de un Servicio de Ortopedia y Traumatología durante la pandemia: 1) continuar la atención de las urgencias, 2) proteger a los pacientes y los profesionales de la salud frente a la transmisión y el contagio, y 3) conservar los recursos disponibles. ${ }^{4}$

Por la fortuna del devenir temporal posterior de los efectos de la pandemia en nuestro país respecto de otras regiones, contamos con el aporte de publicaciones originarias de Asia, Europa y América del Norte, que representaron un aporte vital para el desarrollo de un plan de contingencia que contemple las diferentes áreas de nuestra disciplina. ${ }^{4-11}$ Se adaptaron las recomendaciones disponibles a la fecha publicadas por sociedades científicas internacionales e instituciones de prestigio. ${ }^{12-16}$ Dicha información permitió, en tiempo y forma, adaptar nuestra labor como Servicio de Ortopedia y Traumatología de un hospital público de la Ciudad Autónoma de Buenos Aires. Ya hemos publicado el proceso de elaboración de nuestra estrategia. ${ }^{17}$

El objetivo principal de este estudio fue comparar la actividad clínico-quirúrgica de nuestro Servicio de Ortopedia y Traumatología antes de la instauración del plan de contingencia frente a la pandemia de la COVID-19 y durante este. Los datos obtenidos son relevantes para establecer antecedentes que permitan guiar nuestro desempeño ante la segunda ola de casos y futuras contingencias semejantes.

\section{MATERIALES Y MÉTODOS}

Se realizó un estudio analítico observacional longitudinal ambispectivo de los pacientes atendidos por el Servicio de Ortopedia y Traumatología de un hospital del sistema público de salud de la Ciudad Autónoma de Buenos Aires, durante la implementación del plan de contingencia frente a la pandemia de la COVID-19 acorde a los protocolos y lineamientos establecidos por los Ministerios de Salud de la Nación y de la Ciudad Autónoma de Buenos Aires. ${ }^{17}$ El período de estudio abarcó desde abril de 2019 hasta septiembre de 2020, intervalo que involucró el año anterior a la pandemia y el probable pico de casos de la COVID-19, según los reportes epidemiológicos oficiales disponibles cuando se inició el estudio (Figura 1).

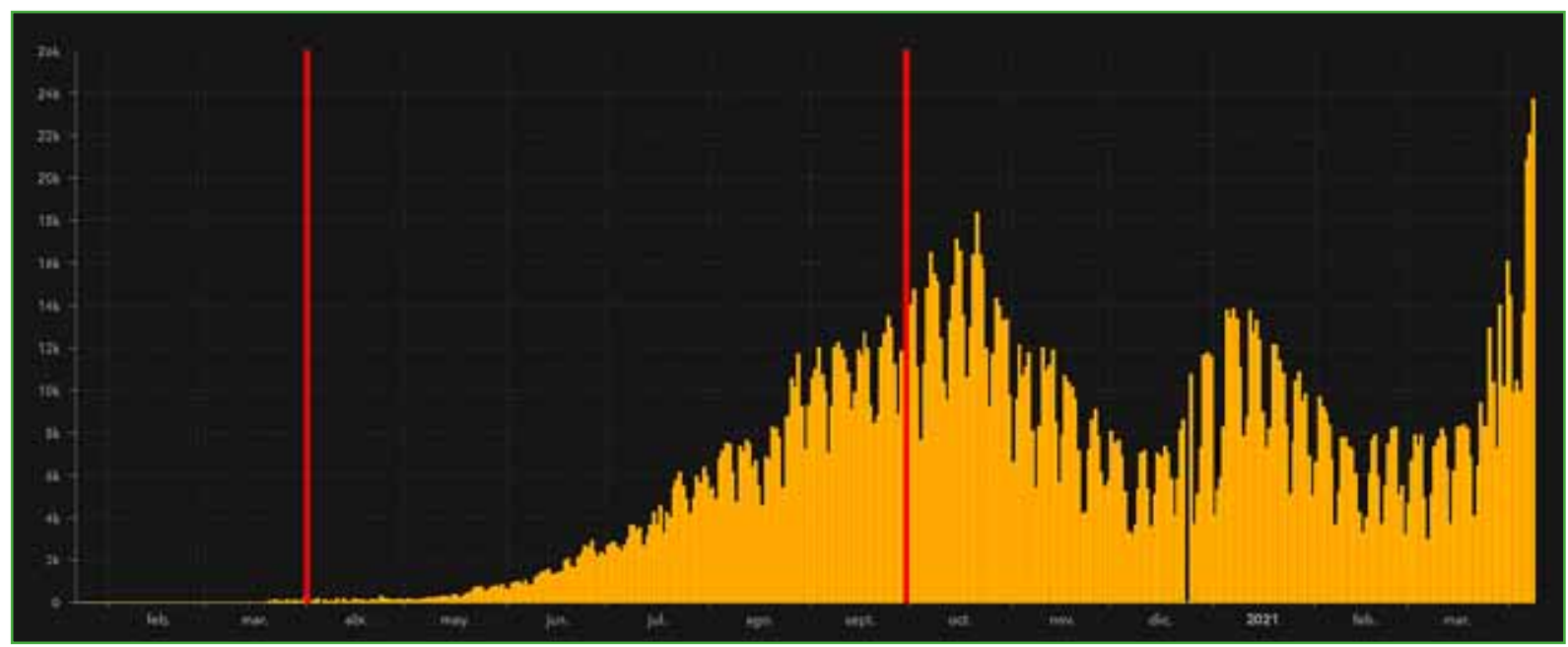

Figura 1. Curva de casos diarios por mes: febrero de 2020-marzo 2021. Período de pandemia considerado: abril-septiembre (entre líneas rojas). Fuente: John Hopkins University. Disponible en: https://www.arcgis.com/apps/opsdashboard/index. html\#/bda7594740fd40299423467b48e9ecf6 
Los criterios de inclusión fueron: registros de pacientes atendidos en el Servicio de Ortopedia y Traumatología y el área de Traumatología del Departamento de Emergencias. Los criterios de exclusión fueron: libros, registros e historias clínicas incompletos o ilegibles en las variables de interés.

Se registraron las variables de estudio tomadas de los datos de historias clínicas, registros administrativos y archivos de nuestra institución. Como variable de resultado primaria se valoró el número total de cirugías realizadas durante el período de estudio. Se dividió a las cirugías en cinco categorías, según la clasificación propuesta por Massey y la American Association of Orthopedic Surgeons, teniendo en cuenta la demora quirúrgica que admiten: emergencias (cirugía dentro de las $24 \mathrm{~h}$ ), urgencias (cirugía dentro de las $48 \mathrm{~h}$ ), urgencias programables (dentro de las 2 semanas), programadas a corto plazo (dentro de los 3 meses), programadas a largo plazo (admite demoras superiores a 3 meses). ${ }^{8}$ La variable se dividió en dos: "urgentes" y "programadas". Se agrupó en "cirugías de urgencia" a las clasificadas como emergencias, urgencias y urgencias programables y en "cirugías programadas" a aquellas programables a corto y largo plazo. La cantidad total de cirugías calculadas involucró el registro de las cirugías de Planta y las cirugías de Guardia que, en este último caso, incluyen cirugías de Guardia mayores y procedimientos menores (cirugías con anestesia local, suturas de heridas).

Se registraron también la cantidad de cirugías por equipo (miembro superior, miembro inferior, pelvis y cadera, rodilla, columna y hombro), la necesidad de internación (internación/ambulatorias) y el promedio de cirugías realizadas por día quirúrgico asignado.

Como variables independientes se evaluaron aquellas relacionadas con el movimiento hospitalario de nuestras salas de internación (Unidades 10 y 11), la actividad en la Guardia y los consultorios externos: ingresos, pases a nuestras unidades de internación, egresos, promedio de días de estancia, porcentaje de ocupación de camas, giro/ cama, pacientes atendidos en la Guardia y turnos asignados en consultorios externos.

Se registraron las actividades no traumatológicas realizadas por traumatólogos durante la pandemia, no incluidas en la comparación, pero de valor para el autor en la descripción del contexto: pacientes atendidos por traumatólogos en el consultorio de febriles e hisopados a cargo de los traumatólogos.

El registro prospectivo de datos comenzó una vez otorgada la aprobación del Comité de Ética de nuestra institución, respetando las normas bioéticas de la Declaración de Helsinki y las normativas vigentes, asegurando la confidencialidad y la seguridad de los datos.

Se confeccionaron formularios de registro para ser completados diariamente y luego realizar la carga semanal en planillas digitales con control mensual de los datos ingresados por un médico a cargo de dicha tarea. Esta información se sumó al registro de datos retrospectivos del período prepandemia. Fue necesaria la participación exhaustiva de médicos de cada Guardia semanal, residentes y médicos de Planta con actividad en quirófano, sala de internación y consultorios externos, que conforman el equipo de investigación.

\section{Comparación}

Las variables de estudio se midieron en dos intervalos de tiempo definidos dentro del período de estudio para su comparación: 1) durante la pandemia: de abril a septiembre de 2020, 2) fuera de la pandemia de la COVID-19 (de abril a septiembre de 2019).

\section{Análisis estadístico}

Las variables categóricas se expresan como número y porcentaje, y se analizaron con la prueba de ji al cuadrado o la prueba de Fisher. Las variables interválicas se describen con media y mediana, según su distribución y sus medidas de dispersión, con desviación estándar (DE) e intervalo intercuartílico 25-75 (IIC). Se realizó la prueba de normalidad de Shapiro-Wilk para estimar el tipo de distribución de las variables cuantitativas. Para la comparación de las variables continuas se utilizaron las pruebas t de Student o U de Mann-Whitney, de acuerdo con la distribución expresada. Se consideró estadísticamente significativo un valor $\mathrm{p}<0,05$. Para el análisis se utilizó el programa SPSS Statics 25. 


\section{RESULTADOS}

Durante el período de la pandemia de la COVID-19 considerado, la actividad quirúrgica disminuyó significativamente, se efectuaron 127 cirugías (media $=21, \mathrm{DE} \pm 2$ ): 104 de Planta (media $=17, \mathrm{DE} \pm 7$ ) y 23 de Guardia (media $=4, \mathrm{DE} \pm 3$ ). La actividad quirúrgica por equipos solo comprendió 35 cirugías de miembro superior, 28 de miembro inferior, 32 de pelvis y cadera, cinco de rodilla, cinco de hombro y una de columna. Estas cifras son significativamente inferiores a las registradas en la actividad habitual de nuestro Servicio antes de la pandemia. La descripción y los resultados obtenidos de la comparación entre los períodos anterior a la pandemia y durante la pandemia se resumen en las Tablas 1-3.

Tabla 1. Cirugías en función del período de trabajo: prepandemia y durante la pandemia

\begin{tabular}{|c|c|c|c|}
\hline \multirow[b]{2}{*}{ Variables } & \multicolumn{2}{|c|}{ Período } & \multirow[b]{2}{*}{$\mathbf{p}^{*}$} \\
\hline & $\begin{array}{c}\text { Prepandemia } \\
\text { (Abril-Septiembre 2019) }\end{array}$ & $\begin{array}{c}\text { Durante la pandemia } \\
\text { (Abril-Septiembre 2020) }\end{array}$ & \\
\hline $\begin{array}{l}\text { Cirugías } \\
\text { n: media (DE)/mediana (IIC) } \\
\text { Totales } \\
\text { Planta } \\
\text { Guardia } \\
\text { Menores } \\
\text { Mayores } \\
\text { De urgencia } \\
\text { Programadas } \\
\text { Con internación } \\
\text { Ambulatorias }\end{array}$ & $\begin{array}{c}\text { 687: } 115(19) / 117(105-126) \\
\text { 253: } 42(13) / 40(31-51) \\
\text { 434: } 72(11) / 76(68-78) \\
\text { 421: } 70(12) / 75(64-77) \\
\text { 4: } 2(1) / 2(1-3) \\
\text { 472: } 79(30) / 92(67-99) \\
\text { 141: } 24(9) / 22(16-27) \\
\text { 202: } 34(9) / 32(28-43) \\
\text { 485: } 81(15) / 84(74-92)\end{array}$ & $\begin{array}{c}\text { 127: } 21(9) / 25(11-28) \\
\text { 104: } 17(7) / 21(10-23) \\
\text { 23: } 4(3) / 3(2-5) \\
\text { 14: } 2(2) / 2(1-3) \\
\text { 9: } 2(1) / 1(1-3) \\
\text { 124: } 21(9) / 25(11-27) \\
\text { 3: } 1(1) / 0(0-1) \\
\text { 93: } 16(8) / 16(11-19) \\
\text { 34: } 6(5) / 6(1-10)\end{array}$ & $\begin{array}{l}0,002 \\
0,002 \\
0,000 \\
0,002 \\
0,358 \\
0,080 \\
0,002 \\
0,080 \\
0,002\end{array}$ \\
\hline $\begin{array}{l}\text { Equipos } \\
\text { n / media (DE)/mediana (IIC) } \\
\text { Miembro superior } \\
\text { Miembro inferior } \\
\text { Pelvis y cadera } \\
\text { Rodilla } \\
\text { Hombro } \\
\text { Columna vertebral }\end{array}$ & $\begin{array}{c}11(6) / 10(6-18) \\
7(3) / 7(5-8) \\
11(5) / 10(7-16) \\
11(5) / 10(7-15) \\
2(1) / 3(1-3) \\
3(1) / 3(2-4)\end{array}$ & $\begin{array}{l}6(3) / 6(3-8) \\
5(2) / 5(4-6) \\
5(3) / 6(3-8) \\
1(1) / 1(0-1) \\
1(1) / 1(0-1) \\
0(0) / 0(0-0)\end{array}$ & $\begin{array}{c}0,73 \\
0,176 \\
0,035 \\
0,002 \\
0,180 \\
0,002\end{array}$ \\
\hline $\begin{array}{l}\text { Cirugías Planta/día } \\
\mathrm{n} / \text { media (DE)/mediana (IIC) }\end{array}$ & $2,7(0,6) / 2,7(2,5-2,8)$ & $1,4(0,3) /, 3(1,2-1,6)$ & 0,000 \\
\hline
\end{tabular}

*Para las variables categóricas se utilizaron la prueba de ji al cuadrado o la prueba de Fisher y, para las variables numéricas, la prueba t de Student o la prueba U de Mann-Whitney, según su distribución.

$\mathrm{DE}=$ desviación estándar, IIC = intervalo intercuartílico. 
Tabla 2. Movimiento hospitalario en función del período de trabajo: prepandemia y durante la pandemia

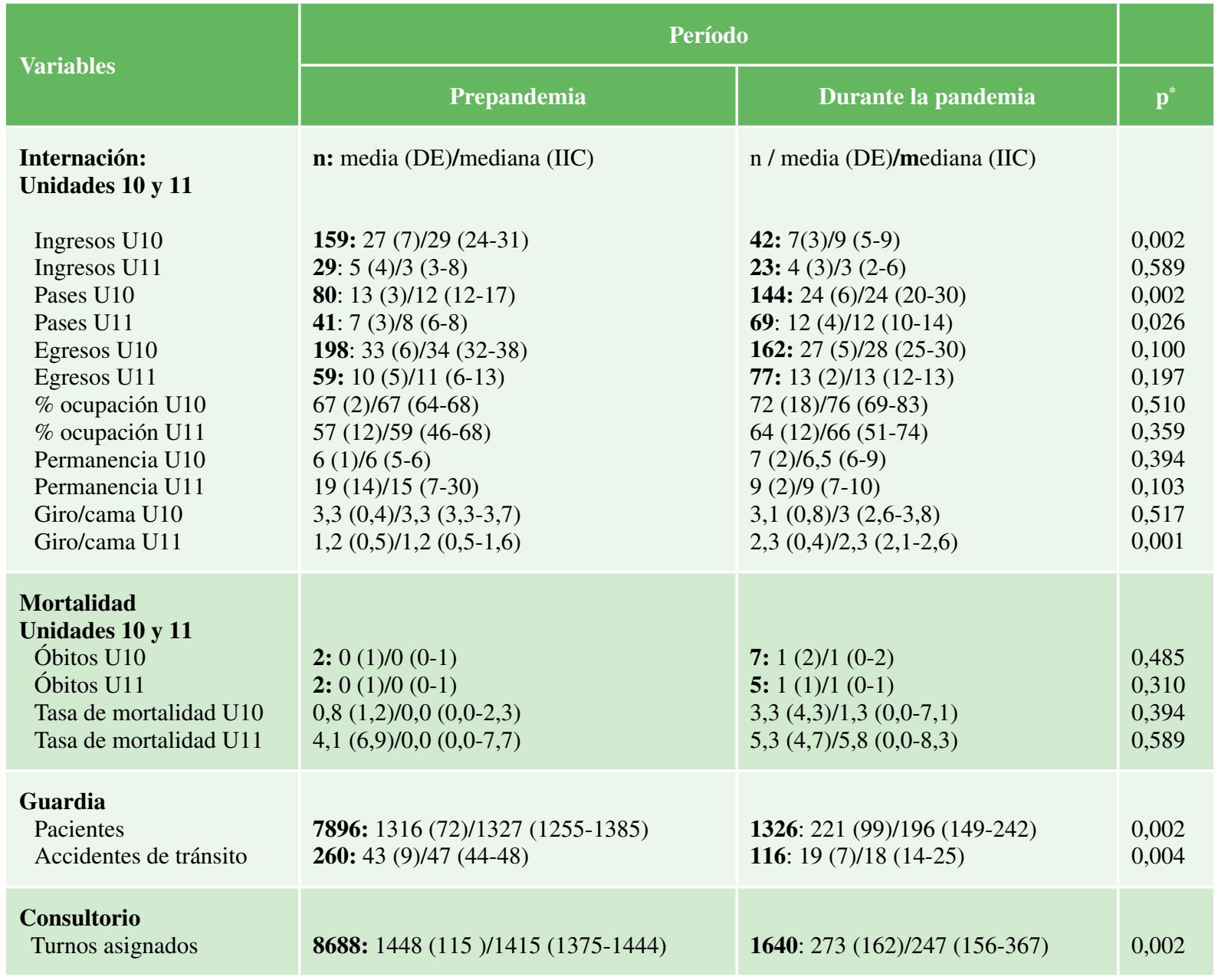

*Para las variables categóricas se utilizaron la prueba de ji al cuadrado o la prueba de Fisher y, para las variables numéricas, la prueba t de Student o la prueba U de Mann-Whitney, según su distribución.

$\mathrm{DE}=$ desviación estándar, IIC = intervalo intercuartílico.

Tabla 3. Tareas no traumatológicas durante la pandemia

\begin{tabular}{|l|c|c|c|c|c|c|c|}
\hline \multirow{2}{*}{ Variables } & \multicolumn{9}{|c|}{ Período } \\
\cline { 2 - 8 } & Abril & Mayo & Junio & Julio & Agosto & Septiembre & Total \\
\hline Pacientes febriles & 3 & 38 & 78 & 175 & 188 & 191 & $673 ;$ media = 112 (DE \pm 83$)$ \\
\hline Hisopados & 2 & 7 & 30 & 68 & 58 & 161 & $326 ;$ media = 54 (DE \pm 59$)$ \\
\hline DE = desviación estándar. & & & & & &
\end{tabular}




\section{Actividad quirúrgica durante la pandemia}

El período de pandemia se asoció significativamente con menos cirugías totales $(\mathrm{p}=0,002)$, cirugías de Guardia $(p=0,000)$ y de Planta $(p=0,002)$. Las cirugías totales se redujeron un $81,5 \%$ con respecto a la etapa prepandemia (prepandemia 685 y pandemia 127). La mayoría de los pacientes requirió internación, las cirugías ambulatorias representaron el $27 \%$ de todas las intervenciones (34 de 127 cirugías) a diferencia del período prepandemia (70\% de cirugías ambulatorias; 485 de 687). Las cirugías programadas se limitaron a tres procedimientos exclusivamente que involucraron el retiro de un tornillo transindesmal $(\mathrm{n}=2)$ y la dinamización de un clavo endomedular $(\mathrm{n}=3)$, todas en el contexto del tratamiento de fracturas agudas. Las cirugías de urgencia representaron el 97\% (124 de 127 cirugías), una diferencia significativa con el período sin pandemia ( $\mathrm{p}=0,080 ; 472$ de 687 cirugías prepandemia; $69 \%)$. Asimismo, el promedio de cirugías por día quirúrgico disponible fue significativamente menor $(\mathrm{p}=0,000)$ (Figura 2).

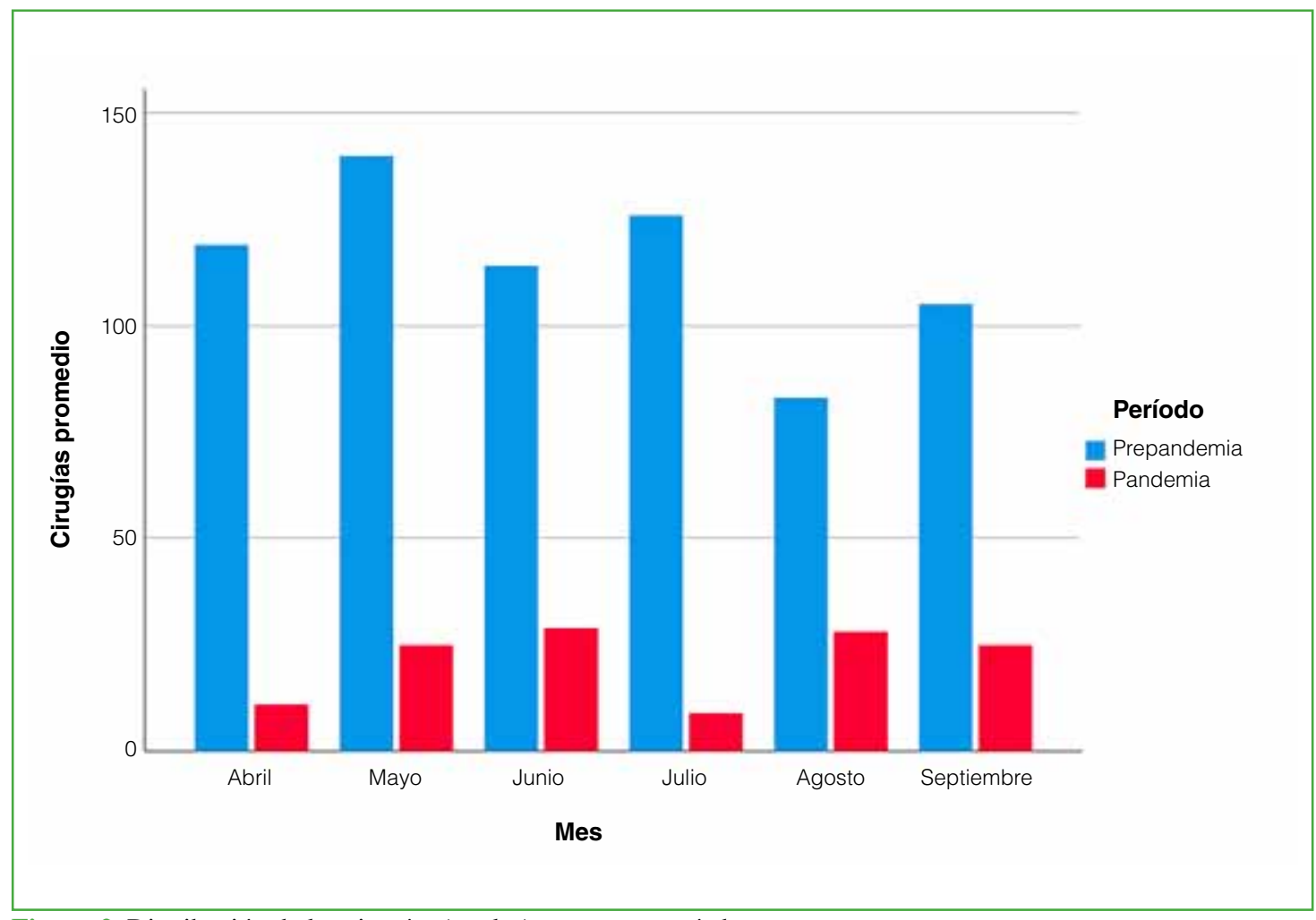

Figura 2. Distribución de las cirugías (totales) por mes y período.

\section{Movimiento hospitalario en las Unidades de Internación}

El movimiento hospitalario durante la pandemia se asoció significativamente con un menor número de "ingresos" (Unidad 10; $p=0,002)$ y un incremento en los "pases" desde otros servicios y la Guardia $(p=0,002)$. No hubo diferencias significativas en el promedio de días de permanencia, pero se optimizó la variable giro/cama en una de las unidades (Unidad 11: giro/cama $\mathrm{p}=0,001$ ). Si bien aumentó el porcentaje de ocupación de camas, no fue estadísticamente significativo con respecto al período prepandemia $(p=0,510 ; p=0,359)$. 


\section{Actividad en la Guardia y los consultorios}

La pandemia se asoció significativamente con menos actividad en nuestra especialidad que se vio reflejada en una menor cantidad de pacientes atendidos (prepandemia: $\mathrm{n}=7896$; media $=1316, \mathrm{DE} \pm 72$; pandemia: $\mathrm{n}=1326$; media $=221, \mathrm{DE} \pm 99 ; \mathrm{p}=0,002$ ), menor atención de accidentes de tránsito (prepandemia: $\mathrm{n}=260$; media $=43$, $\mathrm{DE} \pm$ 9; pandemia: $\mathrm{n}=116$; media $=19, \mathrm{DE} \pm 7 ; \mathrm{p}=0,004)$; una tasa más baja de cirugías, especialmente de procedimientos menores habituales (prepandemia: $\mathrm{n}=421$; media $=70, \mathrm{DE} \pm 12$; pandemia: $\mathrm{n}=14$; media $=2$, $\mathrm{DE} \pm 2 ; \mathrm{p}=0,002)$ (Figura 3).

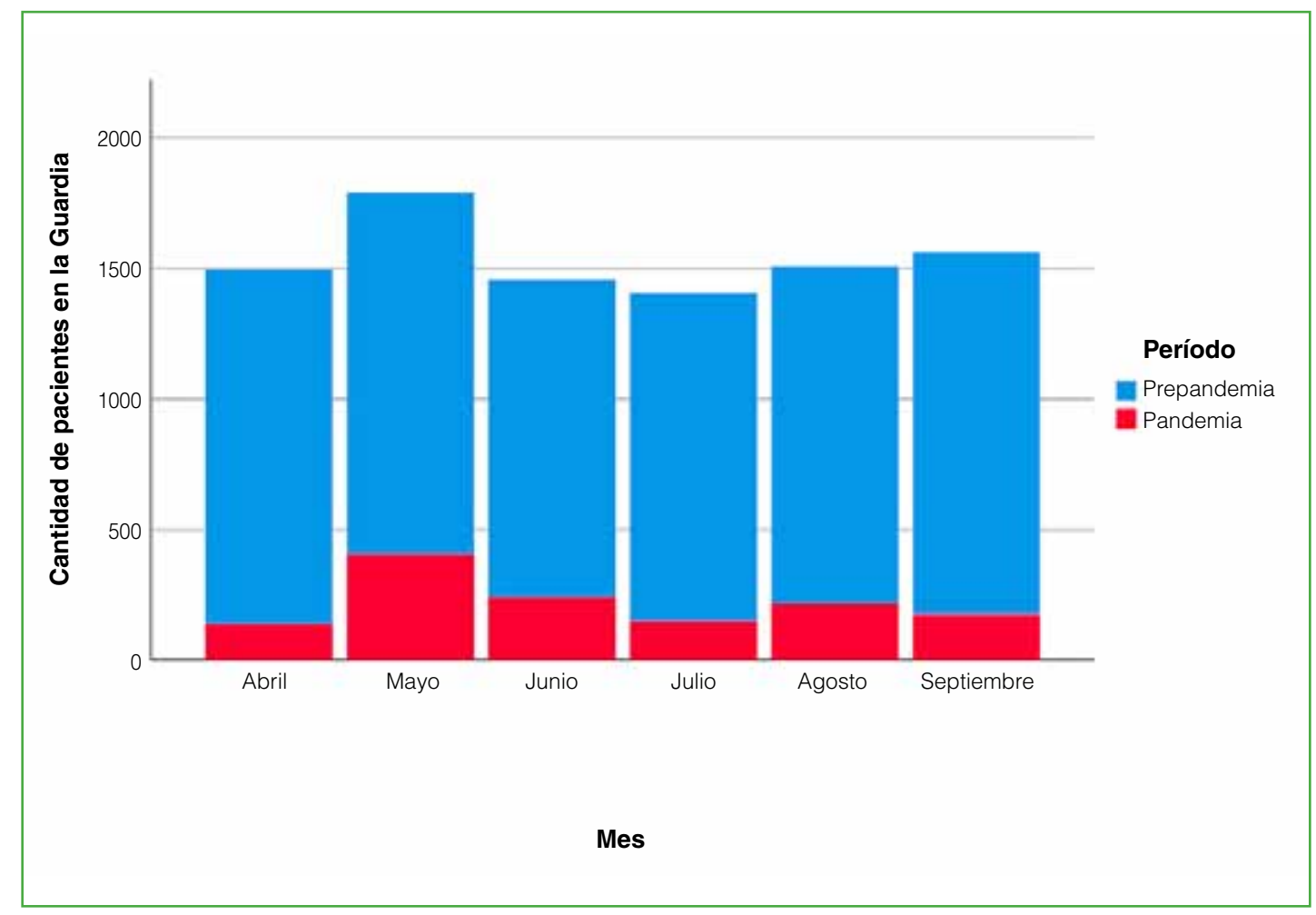

Figura 3. Distribución de los pacientes de Guardia por mes y período.

Asimismo, se asoció con una demanda mucho menor de la atención en consultorios externos, con un 19\% de turnos asignados: programados y espontáneos en comparación con el período prepandemia (prepandemia: $\mathrm{n}=8688$; media $=1448, D E \pm 115$ ); pandemia: $n=1640 ;$ media $=273, D E \pm 162 ; p=0,002$ ) (Figura 4). 


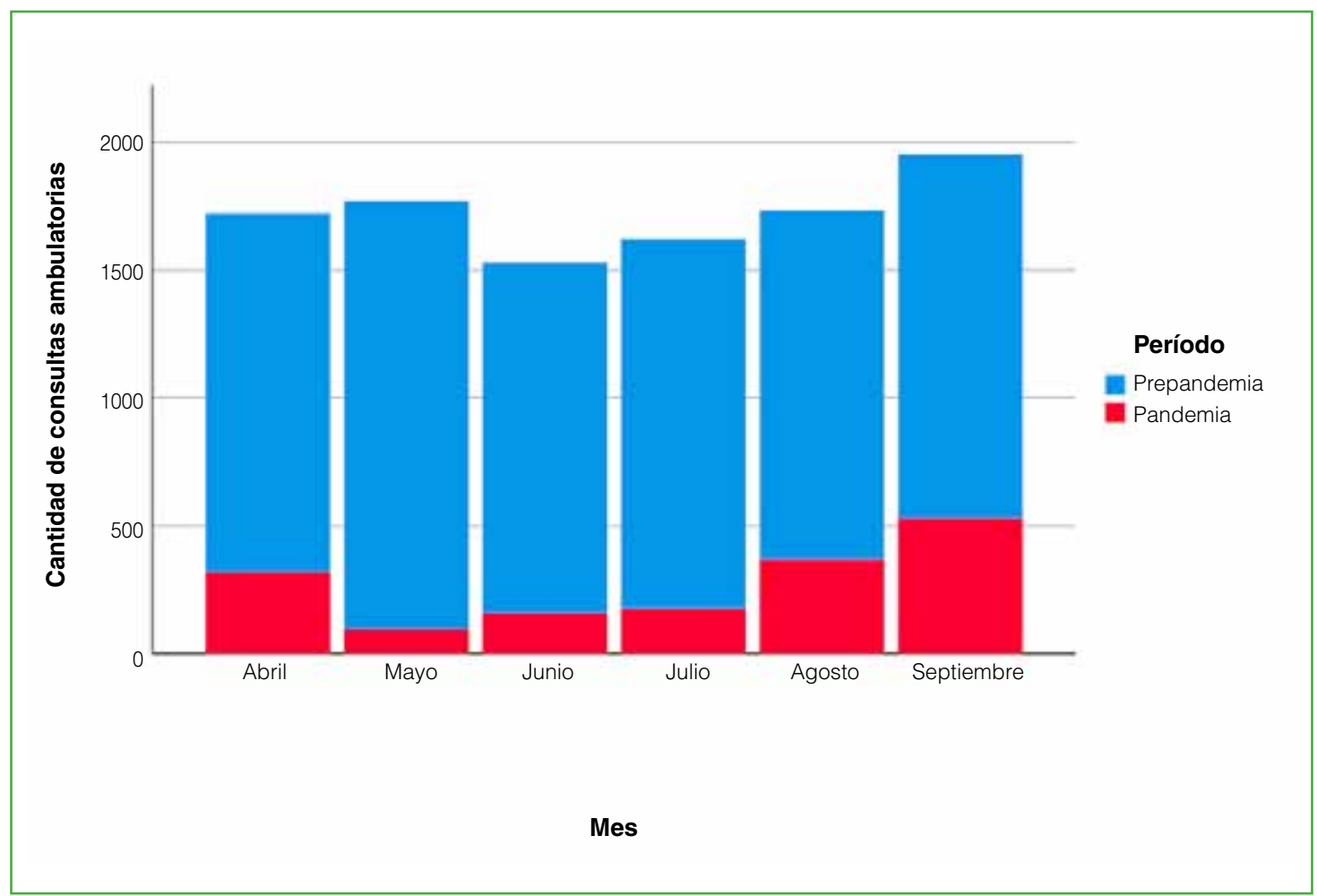

Figura 4. Distribución de las consultas ambulatorias por mes y período.

\section{Actividades no traumatológicas}

Durante la pandemia, se requirió la participación de traumatólogos en áreas no traumatológicas que involucraron: triage en el ingreso hospitalario según el motivo de consulta, atención de 673 pacientes en las Unidades Febriles de Urgencia y llevar a cabo 326 hisopados de casos sospechosos (Tabla 3). En estas tareas, participaron exclusivamente los traumatólogos de Planta. Los residentes y traumatólogos de Guardia por protocolo durante la pandemia realizaron el hisopado nasofaríngeo como estudio antes de la internación en las Unidades 10 y 11, pero dicho número no fue contemplado en el registro (Figura 5).

\section{DISCUSIÓN}

Durante la adaptación de la actividad clínico-quirúrgica del Servicio a la contingencia de la pandemia de la COVID-19, nuestro objetivo principal fue continuar la atención de las urgencias en Ortopedia y Traumatología. No obstante, el contexto epidemiológico en la atención del trauma fue notablemente diferente.

Como se describió en los resultados, la pandemia se asoció significativamente con la reducción de la actividad quirúrgica. La disminución de la tasa de cirugías totales y discriminadas por servicios de Planta y de Guardia podría relacionarse con la menor atención de pacientes en la Guardia (consultas y accidentes de tránsito) y consultorios externos. Sin embargo, también podría estar relacionada con una menor disponibilidad de turnos quirúrgicos por día (disminución del promedio de cirugías por día quirúrgico asignado) y otras variables no evaluadas, como la disponibilidad de anestesiólogos y de camas en terapia intensiva para el posoperatorio. Otras publicaciones similares comunicaron la reducción de la cantidad de cirugías en un 44,2-62,7\%; en nuestro caso, fue del 81,5\%. Las cirugías programadas fueron suspendidas siguiendo los lineamientos del plan de contingencia definido por el Ministerio de Salud de la Ciudad Autónoma de Buenos Aires. De acuerdo con el contexto epidemiológico, durante la primera ola de casos, no fue necesario optar por un manejo ambulatorio precoz de los pacientes quirúrgicos ni modificar los criterios de internación durante la pandemia. 


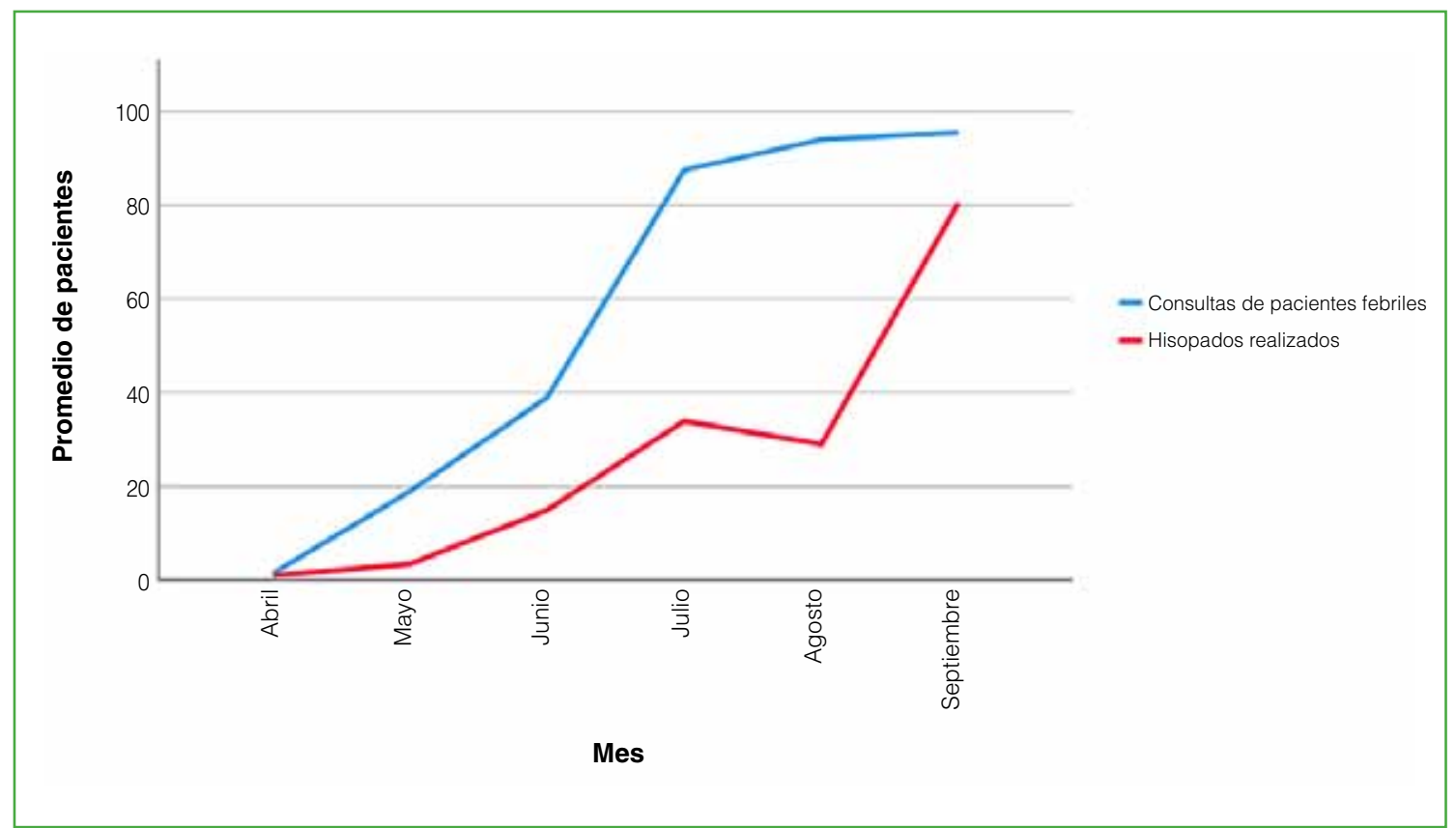

Figura 5. Distribución de hisopados y consultas de pacientes febriles realizados por traumatólogos según mes y período.

La atención en el Servicio de Urgencias disminuyó significativamente: menos atención de pacientes por accidentes de tránsito, procedimientos quirúrgicos y consultas. Esto podría relacionarse, siguiendo el razonamiento de experiencias internacionales similares, ${ }^{18-22}$ con la disminución en la circulación por la cuarentena obligatoria y su apertura progresiva, el temor a la consulta por el riesgo de contagio y la menor actividad laboral presencial.

La demanda en consultorios externos fue mucho más baja debido a que se suspendieron los turnos espontáneos y se restringieron las agendas. Esto se hizo de forma escalonada, la restricción inicial fue máxima, se cerraron las agendas personales de subespecialidades y se dispusieron turnos generales de atención. Las consultas se limitaron al seguimiento de pacientes recién operados y con cuadros urgentes. Transcurridos los primeros meses de la pandemia, el Gobierno de la Ciudad Autónoma de Buenos Aires instauró como complemento un sistema de atención de teleconsultas no incluido en nuestro registro. Otros reportes epidemiológicos describen estrategias y sucesos similares y una disminución de las consultas ambulatorias del 29\%; 23,24 nosotros observamos una reducción mucho mayor, recibimos solo el $19 \%$ de las consultas habituales.

Nuestro Servicio dejó de ser una unidad de internación de Ortopedia y Traumatología predominantemente, para permitir la internación de pacientes "sin" COVID de otras especialidades. Este hecho se vio reflejado en el menor número de ingresos de traumatología en nuestras Unidades y el aumento significativo de los pases de Guardia y otras especialidades. De acuerdo con el contexto epidemiológico descrito, si bien aumentó el porcentaje de ocupación de nuestras salas de internación, nuestra capacidad de atención se mantuvo en niveles razonables, lo que nos permitió atender, internar y operar a pacientes con cuadros de urgencia.

Si bien no representa el objetivo principal de nuestro trabajo, se estimó una tasa de mortalidad más alta durante la internación, en el período de pandemia. Esta diferencia no fue estadísticamente significativa y está sesgada por la internación adicional de pacientes no traumatológicos durante el período de estudio.

\section{CONCLUSIONES}

La atención de la enfermedad ortopédica y el trauma musculoesquelético, adaptada a las obligaciones indicadas por el plan nacional y regional de contingencia para la pandemia, fue notoriamente menor que nuestro desempeño habitual. El contexto epidemiológico se caracterizó por la disminución de la frecuencia de accidentes, la menor demanda de pacientes y la suspensión de la atención de los cuadros programados, suspendida por protocolo del Gobierno de la Ciudad. De acuerdo con nuestro registro, trabajamos sin sufrir el colapso de nuestra capacidad 
de atención, pudimos incluir tareas de soporte no traumatológicas. De esta manera, concluimos en que, ante la preocupación local y global vigente por la adaptación de nuestro desempeño, es de vital importancia reconstruir con datos lo actuado para retroalimentar y redefinir estrategias. Por ello, en nuestro caso, creemos que, si bien respondimos adecuadamente a las urgencias de nuestra especialidad, queda pendiente la posibilidad de estratificar y considerar tipos de pacientes con enfermedades pasibles de ser tratadas gradualmente durante la pandemia.

\section{Agradecimientos}

A todos los integrantes del equipo de salud de la División de Ortopedia y Traumatología y el Servicio de Urgencias de nuestra institución, que llevaron a cabo las actividades asistenciales durante el período de estudio y sobre las cuales fue realizado este trabajo.

Conflicto de intereses: Los autores no declaran conflictos de intereses.

ORCID de F. Locaso: https://orcid.org/0000-0003-2248-5582

ORCID de M. A. Pérez: https://orcid.org/0000-0002-2163-0680

ORCID de S. Soto Arriaga: https://orcid.org/0000-0002-0201-6394

ORCID de S. Scalambro: https://orcid org/0000-0002-7478-0041

ORCID de A. A. Salinas: https://orcid.org/0000-0002-3137-2048

ORCID de M. Riva: https://orcid.org/0000-0003-2494-8456

ORCID de A. Elorza: https://orcid.org/0000-0002-4877-3083

ORCID de D. Mansilla: https://orcid.org/0000-0002-0025-1251
ORCID de F. Molina: https://orcid.org/0000-0002-3747-044X

ORCID de J. Urrica: https://orcid.org/0000-0001-5939-4014

ORCID de G. Ricciardi: https://orcid.org/0000-0002-6952-7260

ORCID de S. Manterola: https://orcid. org/0000-0003-4194-1563

ORCID de I. G. Garfinkel: https://orcid.org/0000-0001-9557-0740

ORCID de G. G. Carrioli: https://orcid.org/0000-0003-4160-9712

ORCID de D. O. Ricciardi: https://orcid.org/0000-0002-1396-9115

\section{BIBLIOGRAFÍA}

1. Zhu N, Zhang D, Wang W, Li X, Yang B, Song J, et al, China Novel Coronavirus Investigating and Research Team. A novel coronavirus from patients with pneumonia in China, 2019. N Engl J Med 2020;382(8):727-33. https://doi.org/10.1056/NEJMoa2001017

2. World Health Organization Press Conference. The World Health Organization (WHO) has officially named the disease caused by the novel coronavirus as COVID-19. [Consulta: 11 de febrero, 2020] Disponible en: https://www.who.int/emergencies/diseases/novel-coronavirus-2019

3. Emergencia Sanitaria. Decreto 260/2020 DECNU-2020-260-APN-PTE - Coronavirus (COVID-19). Boletín Oficial de la República Argentina, edición No 34.327 de la Primera Sección del jueves 12 de marzo de 2020. Disponible en: https://www.boletinoficial.gob.ar/detalleAviso/primera/5217883/20200312?suplemento=1

4. Chang Liang Z, Wang W, Murphy D, Po Hui JH. Novel coronavirus and orthopaedic surgery: early experiences from Singapore. J Bone Joint Surg Am 2020;102(9):745-9. https://doi.org/10.2106/JBJS.20.00236

5. Rodrigues-Pinto R, Sousa R, Oliveira A. Preparing to perform trauma and orthopaedic surgery on patients with COVID-19. J Bone Joint Surg Am 2020;102(11):946-50. https://doi.org/10.2106/JBJS.20.00454

6. Ashford RU, Nichols JS, Mangwani J. Annotation: The COVID-19 pandemic and clinical orthopaedic and trauma surgery. J Clin Orthop Trauma 2020;11(3):504-5. https://doi.org/10.1016/j.jcot.2020.04.002

7. Liang ZC, Ooi S. COVID-19: A Singapore orthopedic resident's musings in the Emergency Department. Acad Emerg Med 2020;27(4):349-50. https://doi.org/10.1111/acem.13970

8. Massey PA, McClary K, Zhang AS, Savoie FH, Barton RS. Orthopaedic surgical selection and inpatient paradigms during the coronavirus (COVID-19) pandemic. J Am Acad Orthop Surg 2020;28(11):436-50. https://doi.org/10.5435/JAAOS-D-20-00360

9. Randelli PS, Compagnoni R. Management of orthopaedic and traumatology patients during the coronavirus disease (COVID-19) pandemic in northern Italy. Knee Surg Sports Traumatol Arthrosc 2020;28(6):1683-9. https://doi.org/10.1007/s00167-020-06023-3

10. Prada C, Chang Y, Poolman R, Johal H, Bhandari M. Best practices for surgeons-COVID-19 evidence-based scoping review. A unifying report of global recommendations. [Consulta: 4 de abril, 2020] Disponible en: https://aaot.org.ar/wp-content/uploads/2020/04/OE-Best-Practices-for-Surgeons-COVID-19-Evidence-Based-ScopingReview.pdf_compressed1.pdf 
11. Vaccaro AR, Getz CL, Cohen BE, Cole BJ, Donnally CJ 3rd. Practice management during the COVID-19 pandemic. J Am Acad Orthop Surg 2020;28(11):464-70. https://doi.org/10.5435/JAAOS-D-20-00379

12. SECOT. Recomendaciones generales de la Sociedad Española de Cirugía Ortopédica y Traumatología frente a la COVID-19. 2020. [Consulta: 24 de marzo, 2020] Disponible en: https://www.secot.es/media/docs/covid19

13. NHS England and NHS Improvement. Clinical guide for the management of trauma and orthopaedic patients during the coronavirus pandemic. [Consulta: 14 de abril, 2020] Disponible en:

https://www.england.nhs.uk/coronavirus/wp-content/uploads/sites/52/2020/03/C0274-Specialty-guide-Orthopaedictrauma-v2-14-April.pdf

14. British Orthopaedic Association. Management of patients with urgent orthopaedic conditions and trauma during the coronavirus pandemic. [Consulta: 13 de abril, 2020] Disponible en: https://www.boa.ac.uk/resources/covid-19-boasts-combined.htm

15. COVID-19 Guidelines for Triage of Orthopaedic Patients. American College of Surgeons. [Consulta: 4 de abril, 2020] Disponible en: https://www.facs.org/covid-19/clinical-guidance/elective-case/orthopaedics

16. Guidance for surgeons working during the COVID-19 pandemic from the Surgical Royal Colleges of the United Kingdom and Ireland. [Consulta: 20 de marzo, 2020] Disponible en: https://www.rcseng.ac.uk/coronavirus/joint-guidance-for-surgeons-v1/

17. Ricciardi GA, Pérez MA, Scalambro S, Ricciardi G, Carrioli GG, Locaso F, et al. Propuesta para formular una estrategia de contingencia en Ortopedia y Traumatología frente a la pandemia de COVID-19. Rev Asoc Arg Ortop Traumatol 2020;85(3):283-94. https://doi.org/10.15417/issn.1852-7434.2020.85.3.1106

18. Andreozzi V, Marzilli F, Muselli M, Previ L, Cantagalli MR, Princi G, et al. The impact of COVID-19 on orthopaedic trauma: A retrospective comparative study from a single university hospital in Italy. Orthop Rev 2021;12(4):8941. https://doi.org/10.4081/or.2020.8941

19. Battiato C, Berdini M, Luciani P, Gigante A. Impact of coronavirus disease 2019 (COVID-19) on the epidemiology of orthopedics trauma in a region of central Italy. Injury 2020;51(12):2988-9. https://doi.org/10.1016/j.injury.2020.09.044

20. Maniscalco P, Poggiali E, Quattrini F, Ciatti C, Magnacavallo A, Caprioli S, et al. The deep impact of novel CoVID-19 infection in an Orthopedics and Traumatology Department: the experience of the Piacenza Hospital. Acta Biomed 2020;91(2):97-105. https://doi.org/10.23750/abm.v91i2.9635

21. DiFazio LT, Curran T, Bilaniuk JW, Adams JM, Durling-Grover R, Kong K, et al. The impact of the COVID-19 pandemic on hospital admissions for trauma and acute care surgery. Am Surg 2020;86(8):901-3. https://doi.org/10.1177/0003134820939904

22. Alyami AH, Alyami AA, AlMaeen BN. Impact of COVID-19 on orthopedic surgery: Experience from Saudi Arabia. Ann Med Surg (Lond) 2020;56:61-3. https://doi.org/10.1016/j.amsu.2020.05.048

23. Maryada VR, Mulpur P, Guravareddy AV, Pedamallu SK, Vijay Bhasker B. Impact of COVID-19 pandemic on orthopaedic trauma volumes: a multi-centre perspective from the State of Telangana. Indian J Orthop 2020;54(Suppl 2):1-6. https://doi.org/10.1007/s43465-020-00226-Z

24. Wong J, Cheung K. Impact of COVID-19 on Orthopaedic and Trauma Service: An epidemiological study. J Bone Joint Surg Am 2020;102(14):e80. https://doi.org/10.2106/JBJS.20.00775 